\section{Inadvertent toxic drug reaction in the management of atrial fibrillation}

\section{Susannah K Leaver ${ }^{1} \quad$ Timothy B L Ho}

$J$ R Soc Med 2006;99:149-150

When changing medications for atrial fibrillation, sufficient time should be allowed for the elimination of drugs being stopped in order to prevent inadvertent drug interactions.

\section{CASE HISTORY}

A 64-year-old woman presented with a 3-day history of cough, breathlessness and vague abdominal pain. She had appeared well that morning when woken by her husband in order to give her morning medication. He returned 45 minutes later to find the patient semi-conscious. The patient was known to suffer with atrial fibrillation, was awaiting cardioversion, and had been on long-term sotalol, digoxin and warfarin. On the previous day, she had complained of increasing breathlessness and was seen by her general practitioner, who noted that she was in fast atrial fibrillation at 140 beats/min (bpm). He advised stopping the digoxin and sotalol immediately and prescribed verapamil $80 \mathrm{mg}$ three times a day, co-amilofruse and perindopril. These were started on the morning of admission.

The initial examination revealed a Glasgow coma score (GCS) of $14 / 15$, heart rate was $50 \mathrm{bpm}$ atrial fibrillation, blood pressure $120 / 80 \mathrm{mmHg}$ on the left arm and 90/ $60 \mathrm{mmHg}$ on the right, with normal heart sounds and a clear chest. Her respiratory rate was $22 \mathrm{bpm}$ and she was apyrexial. There was some mild upper abdominal tenderness with audible bowel sounds but no palpable abdominal aortic aneurysm. A chest radiograph showed cardiomegaly, but clear lung fields and no air under the diaphragm. An electrocardiogram (ECG, Figure 1) demonstrated slow atrial fibrillation, but no evidence of acute ischaemia or infarction. Arterial blood gases revealed a compensated metabolic acidosis $\left(\mathrm{pH} 7.40, \mathrm{PO}_{2} 31.9 \mathrm{kPa}\right.$ $\mathrm{PCO}_{2} 2.04 \mathrm{kPa} \mathrm{HCO}_{3} 9.8 \mathrm{mmol} / \mathrm{L}$, lactate $5.8 \mathrm{mmol} / \mathrm{L}$ ). A digoxin level at this time was sub-therapeutic at $0.9 \mathrm{nmol} / \mathrm{L}$ (normal range $1-2 \mathrm{nmol} / \mathrm{L}$ ) and the electrolyte results were as follows: sodium $136 \mathrm{mmol} / \mathrm{L}$, potassium

\footnotetext{
${ }^{1}$ Specialist Registrar, ${ }^{2}$ Consultant Chest Physician, Knight Centre for Cystic Fibrosis, Frimley Park Hospital NHS Foundation Trust, Camberley, Surrey GU16 7UJ, UK

Correspondence to: $\operatorname{Dr}$ T B L Ho

E-mail: timothy.ho@fph-tr.nhs.uk
}

$5.5 \mathrm{mmol} / \mathrm{L}$, urea $11.1 \mathrm{mmol} / \mathrm{L}$, creatinine $161 \mu \mathrm{mol} / \mathrm{L}$. Her INR (international normalized ratio) was 4.7, consistent with her being on warfarin. The rest of her haematology was unremarkable. Liver function tests were mildly elevated and C-reactive protein (CRP) and troponin T were normal. Over a 40-min period the patient's GCS deteriorated to $9 / 15$. Her blood pressure dropped to 60/ $30 \mathrm{mmHg}$ associated with peripheral mottling and anuria. No femoral or distal pulses could be felt. Fluid and inotropic resuscitation was started. An urgent thoracic and abdominal computed tomogram (CT) was performed to exclude an aortic dissection. Bowel ischaemia secondary to an embolus was considered but deemed unlikely given that she was on warfarin with a therapeutic INR. We thought about an atypical respiratory infection as her initial presentation was with a dry cough, shortness of breath and vague abdominal discomfort; however her white cell count and CRP were normal. Cardiogenic shock secondary to myocardial infarction was another possibility but again considered unlikely as there were no ischaemic ECG changes.

The CT scan was unremarkable. The patient remained hypotensive and bradycardic in slow atrial fibrillation, despite escalating intravenous adrenaline doses. A repeat blood gas revealed a worsening metabolic acidosis. An external cardiac pacemaker was connected to the patient. Her heart rate went up to $70 \mathrm{bpm}$ and the blood pressure transiently rose to a systolic of $90 \mathrm{mmHg}$. The possibility of an iatrogenic cause of the hypotension was now considered as a result of an interaction between sotalol, digoxin and verapamil. An intravenous bolus of $10 \mathrm{~mL}$ of $10 \%$ calcium chloride was given with no response. A similar result occurred with $600 \mu \mathrm{g}$ of intravenous atropine and $10 \mathrm{mg}$ of intramuscular glucagon. An intravenous temporary pacing wire was inserted and set at a rate of $100 \mathrm{bpm}$. Over a 6hour period the patient's blood pressure stabilized and her GCS reverted to normal. Her heart rate increased to greater than $100 \mathrm{bpm}$, atrial fibrillation. Amiodarone was introduced to control this. Despite being initially oligoanuric and requiring temporary haemodialysis, she made a full recovery. The patient remains well.

\section{DISCUSSION}

Atrial fibrillation remains the most common chronic cardiac arrhythmia. ${ }^{1,2}$ Its management is widely considered and a number of treatment strategies now exist. ${ }^{3}$ This case illustrates how commonly used pharmacological regimens can be potentially fatal. Digoxin, verapamil and sotalol are all recognized treatments for atrial fibrillation. Digoxin inhibits the $\mathrm{Na}^{+/} \mathrm{K}^{+}$ATPase pump and reduces the ventricular rate by decreasing atrioventricular (AV) node conduction. Verapamil is a calcium channel antagonist and 


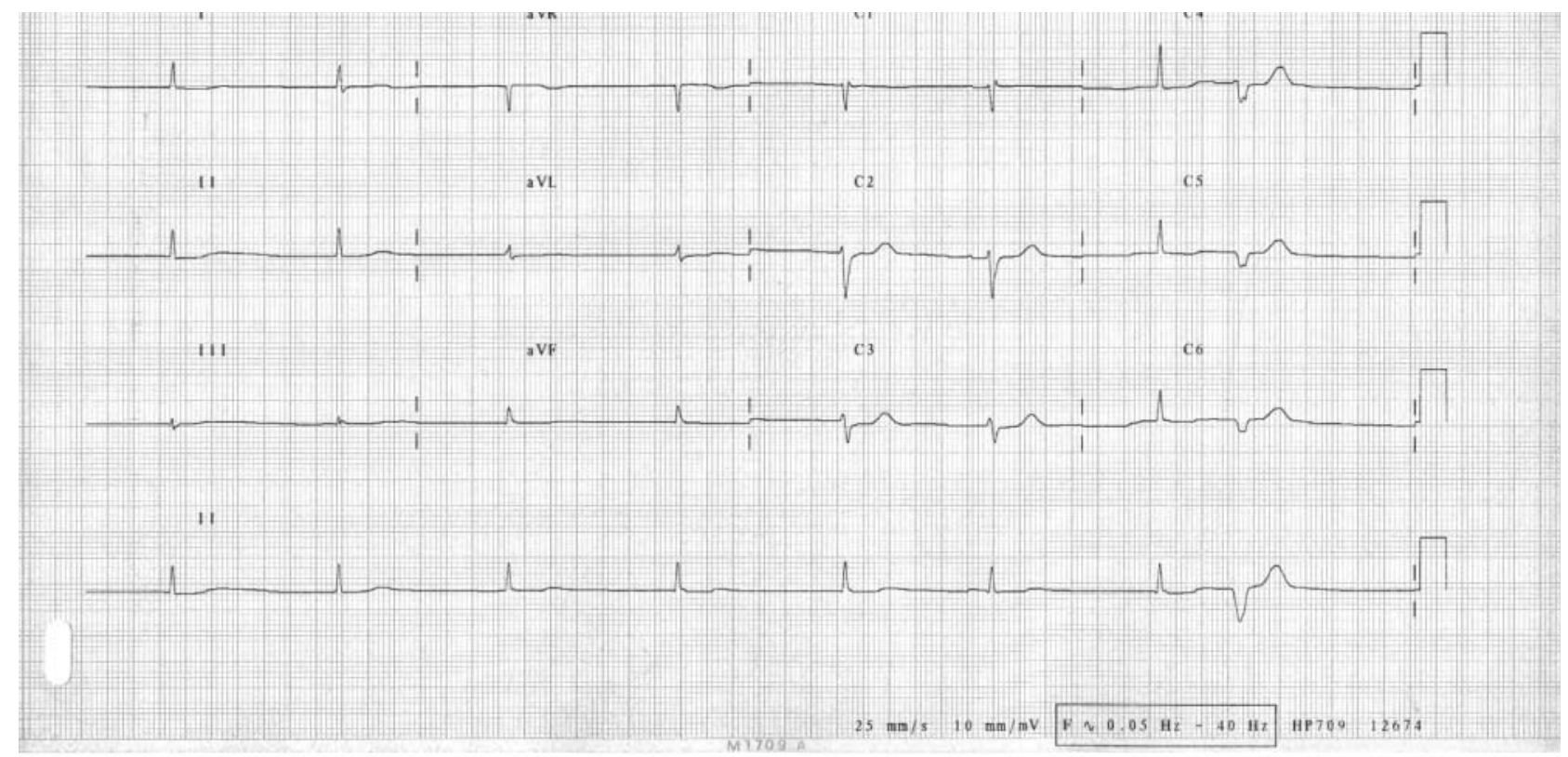

Figure1 An electrocardiogram demonstrating slow atrial fibrillation but no evidence of acute ischaemia or infarction.

has an anti-arrhythmic effect (class IV) by depressing AV node conduction. Sotalol is both a class II $(\beta$-adrenergic blocker) and class III (prolongs the duration of the cardiac action potential) anti-arrhythmic and also slows conduction across the AV node. Therefore the co-prescription of verapamil, $\beta$-blockers, such as sotalol, and digoxin is usually avoided to prevent potent inhibition of the atrioventricular node. In this case there was no intent to use these agents together. However, it is likely that they inadvertently had a synergistic effect. Verapamil was introduced only 12 hours after digoxin and sotalol were stopped. Both digoxin and sotalol are renally excreted. ${ }^{2,4}$ Digoxin has an elimination half-life of 2 days in patients with normal renal function and sotalol an elimination half-life of 12 hours. There is also a direct link between declining elimination rate of these drugs and falling renal function. The patient had demonstrable renal impairment on admission, potentially increasing the effect of these drugs. In addition, verapamil is known to increase the plasma levels of digoxin.

Specific antagonists to these agents were administered in order to improve the patient's haemodynamic status. Calcium chloride was given in an attempt to reverse the effects of verapamil and also for its intrinsic vasopressor effects. ${ }^{5}$ Glucagon was given as an antidote for the $\beta$-blocker. Neither had a significant effect. Fab antibodies to digoxin were not administered, as the digoxin level was sub-therapeutic. This may in fact account for the patient's fast atrial fibrillation when seen by her GP.

An intravenous adrenaline infusion was also ineffective despite being a direct stimulant of $\beta-1$ receptors in the heart. This probably reflected the potent synergistic effect of verapamil, sotalol and digoxin. This and the patient's habitus may also account for the failure of transcutaneous pacing. Indeed, it was only with intracardiac temporary pacing that effective ventricular stimulation was achieved and blood pressure levels restored.

This patient was in persistent atrial fibrillation. She had been treated with sotalol, however a more standard $\beta$-blocker may have been preferable for rate control, as sotalol has been shown to increase the risk of arrhythmias especially in elderly women. ${ }^{6}$

We therefore advise caution with the sequential use of drugs with potentially toxic interactions until sufficient time has passed to ensure adequate elimination of one drug before the introduction of the other. Additionally, physicians should be aware that transcutaneous pacing may not be effective in some patients and in this situation temporary transvenous pacing is indicated.

\section{REFERENCES}

1 Singh BN, Singh SN, Reda DJ, et al. Amiodarone versus sotalol for atrial fibrillation. N Engl J Med 2005;352:1861-72

2 Deneer VH, Borgh MB, Kingma JH, Lie AHL, Brouwers JR. Oral antiarrhythmic drugs in converting recent onset atrial fibrillation. Pharm World Sci 2004;26:66-78

3 Wyse DG, Waldo AL, DiMarco JP, et al. A comparison of rate control and rhythm control in patients with atrial fibrillation. $N$ Engl $\mathrm{J} \mathrm{Med}$ 2002;347:1825-33

4 Warrell D, Cox TM, Firth JD, Benz EJ. Oxford Textbook of Medicine. Oxford: Oxford University Press, 2004

5 Sakurai H, Kei M, Matsubara K, et al. Cardiogenic shock triggered by verapamil and atenolol: a case report of therapeutic experience with intravenous calcium. Jpn Circ J 2000;64:893-6

6 Mickel M, Mitchell LB, Nelson JD, et al. AFFIRM Investigators. Relationship between sinus rhythm, treatment and survival in the Atrial Fibrillation Follow-Up Investigation of Rhythm Management (AFFIRM) Study. Circulation 2004;109:1509-13 